\title{
Expanded downscaling for generating local weather scenarios
}

\author{
Gerd Bürger* \\ Potsdam Institut für Klimafolgenforschung, Telegrafenberg, D-14412 Potsdam, Germany
}

\begin{abstract}
In an attempt to reconcile the capabilities of statistical downscaling and the demands of ecosystem modeling, the technique of expanded downscaling is introduced. Aimed at use in ecosystem models, emphasis is placed on the preservation of daily variability to the extent that possible climate change permits. Generally, the expansion is possible for any statistical model which is formulated by utilizing some form of regression, but I will concentrate on linear models as they are easier to handle. Linear statistical downscaling assumes that the local climate anomalies are linearly linked to the global circulation anomalies. In expanded downscaling, in contrast, I propose that the local climate covariance is linked bilinearly to the global circulation covariance. This is done by transforming the technique of unconstrained minimization of the error cost function into a constrained minimization problem, with the preservation of local covariance forming the side condition. A general normalization routine is inciuded on the local side in order to perform the downscaling exclusively with normally distributed variables. Application of the expanded operator to the daily, global circulation works essentially like a weather generator. Using observed geopotential height fields over the North Atlantic and Europe gave consistent results for the weather station at Potsdam with 14 measured quantities, even for moisture-related variables. For GCM (general circulation model) scenarios, satisfactory results are obtained when the original variables are normally distributed. If they are not, strong sensitivity even to small input changes cause the normalization to produce large errors. Non-normally distributed variables such as most moisture variables are therefore strongly affected by even slight deficiencies of current GCMs with respect to daily variability and climatology. This marks the limit of applicability of expanded downscaling.
\end{abstract}

KEY WORDS: Statistical downscaling - Climate change Climate impact - Weather generator

\section{INTRODUCTION}

In the context of climate change it has become increasingly urgent to fill the gap between the lowresolution large-scale features of climate models and the high-resolution small-scale features of ecosystems which are, in part, driven by the climate. This discrepancy, however, which applies to the spatial as well as the temporal dimension, creates eminent theoretical as well as practical problems. From the climate side, this has been dubbed the 'downscaling problem', and it addresses the problem of deriving local weather scenarios, for the present and future, from a model run of some given General Circulation Model (GCM).

\footnotetext{
•E-mail: buerger@pik-potsdam.de
}

Such a scenario is certainly not meant as a prediction of future weather processes. Instead it tries to estimate shifts, and study the sensitivity, of statistical parameters such as monthly or annual means at a given location together with the variability about them. Unfortunately, many quantities exhibit large spatial variability in their first 2 statistical moments, most notably precipitation, a quantity which is essential to almost all ecosystems. As another obstacle, non-normality of the variables comes into play such that the use of those moments itself is in question. In that case one does not even know what might be important to model. For example, precipitation, which involves both long dry spells and heavy rainfall events, is far from being normal. But exactly those extreme phenomena have the greatest impact. 
The measurements at a weather station are determined by an interaction between the global circulation pattern, $G$, the regional weather elements, $R$, and the local microclimate of that station, $\mathcal{L}$. So far, a nested dynamical approach (cf. e.g. Giorgi 1990) which couples all 3 weather aspects is far from being completed, the main obstacle being the parameterizations at the interfaces. The calibration of these model parameters based on empirical knowledge is particularly difficult as they usually enter the equations non-linearly.

There are quite a few methods that empirically estimate $\mathcal{L}$ directly from $G$, thereby giving up the ambitious dynamical approach. By utilizing already obtained observations, $g$ and $\mathcal{l}$, of $\mathcal{G}$ and $\mathcal{L}$ quantities, respectively, one attempts to establish a direct mathematical relation of the form

$$
l=f(g)
$$

between the local and global weather patterns, leaving out the regional structure $R$ completely. For example, if $g$ represents a summer high-pressure system over the Baltic Sea it is expected that that day's weather in Central Europe is quite sunny and dry, adding an appropriate entry $t$ to a given weather station record. These approaches can be divided into 2 groups, linear and nonlinear techniques. The latter almost always utilize some finite set of what is known as Großwetterlagen and formulate submodels for each element. Among them are those of Bardossy \& Plate (1991), who fit an Autoregressive Moving Average (ARMA) model to daily precipitation records; those of Wilson et al. (1992), who use Empirical Orthogonal Function (EOF) techniques to define the weather regimes; and the Classification and Regression Tree (CART) scheme, which is applied by Hughes et al. (1993) to distinguish between dry and wet periods. The CART scheme and the so-called 'analog' method are applied by Zorita et al. (1995) for the design of rainfall generators. Neural nets, which are currently applied to almost all phenomena, especially nonlinear ones, might also prove valuable in addressing the downscaling problem; see Elsner \& Tsonis (1992) for a thorough account. The fact that many of these techniques are applied to precipitation records, and most notably to the daily time scale, results from the highly nonlinear and particularly regime-like character of this quantity. One of the greater pitfalls of the nonlinear approach is the difficulty of finding stable estimates of the model parameters which have to be fitted. Or, as seen from the reverse side: the output of nonlinear models very often depends too sensitively on the exact value of their parameters. By definition, this sensitivity is largely reduced in linear models. The only thing one has to care about, therefore, is ensuring that the number of estimated model parameters stays within a certain bound given by the number of degrees of freedom of the training set.

In this study I try to assess the statistical downscaling methods in some generality, but with special emphasis on possible applications. The phrase 'statistical downscaling' is used here to mean any method which determines the transfer function $f$ in Eq. (1) by applying some form of least-squares regression technique to the set of parameters in $f$, and $f$ is taken from a predefined functional class. For the class of linear functions it has been shown in a number of studies (von Storch et al. 1993, Werner \& Storch 1993) that one can find quite stable relations between seasonal atmospheric circulation and local weather anomalies, with correlations going as high as $80 \%$ for quantities such as temperature or pressure. To apply these findings to ecosystem models one has to derive, in a second step, daily scenarios which are built upon those mean values, mostly by using tools such as a weather generator

At first sight, there is no reason to restrict attention to monthly or seasonal means and not to downscale daily values directly. But here one faces the fact that with weak correlations the variability of the downscaled process is reduced accordingly, and many ecosystems require a great deal of daily variability as a driving force. In particular, there seems to be no way of modeling daily precipitation correctly, where the weak correlation damps the variability to a small fraction of what is realistic. In this context, another drawback of statistical downscaling which obeys Eq. (1) becomes evident: If the transfer function $f$ is not too nonlinear, the modeled quantity is bound to have essentially the same statistical distribution as the forcing field. But the statistical distribution of the forcing field is primarily normal when one uses circulation variables such as pressure or geopotential height.

This study presents work that pursues both directions mentioned above: I shall formulate a method of applying downscaling techniques directly to obtain daily values, even for quantities that have daily distributions which are far from normal.

\section{LINEAR STATISTICAL DOWNSCALING}

In the simplest case, statistical downscaling models the local process, $l$, using a linear expression of the form

$$
\hat{\imath}=\mathbf{L} \mathscr{g}
$$

with a suitably chosen matrix $\mathbf{L}$. It is assumed that $g$ and ( are given as anomalies about some previously determined (daily, monthly, annual) mean values. It is desirable that the model error $\mathbf{L} g$ - / or, more exactly, the 
model error covariance become as small as possible. Its size can be measured e.g. by the cost function:

$$
\Gamma(\mathbf{L})=\operatorname{tr}\left(\langle(\mathbf{L} g-l)\rangle\langle(\mathbf{L} g-l)\rangle^{\top}\right)
$$

where \langle\rangle denotes expectation). Let us denote the crosscovariance between 2 processes $x$ and $y$ by $\mathbf{C}_{x y}$ (and $\mathbf{C}_{x}$ for $x=y$ ). If, for a sufficiently long time span, $g$ and $f$ are given through observations then the statistical estimates of $\mathbf{C}_{i g}, \mathbf{C}_{i}$, and $\mathbf{C}_{\eta}$ are quite reliable. The cost function can then be expressed as

$$
\Gamma(\mathbf{L})=\operatorname{tr}\left(\mathbf{L C}_{g} \mathbf{L}^{\top}-\mathbf{L C}_{g l}-\mathbf{C}_{g g} \mathbf{L}^{\top}-\mathbf{C}_{l}\right)
$$

The condition of a local extreme, $\partial \Gamma / \partial \mathbf{L}=0$, gives the well-known linear regression solution

$$
\mathbf{L}=\mathbf{C}_{l g} \mathbf{C}_{g}^{-1}
$$

With $n$ global and $m$ local variables, $\mathrm{L}$ is an $m \times n$ matrix. Since the global dimension is usually quite large it is recommended that reduction techniques such as EOF filtering be used before calculating Eq. (5). A further reduction can be achieved by considering a singular value decomposition (SVD) of the matrix $\mathbf{L}$ with an appropriate selection of a few significant singular vectors. Those vectors give the dominant physical mechanism of the impact of $G$ on $L$. A related method involves using Canonical Correlation Analysis (CCA, see Barnett 1991) where one maximizes the correlation between the loadings of 2 fields and uses this correlation for the prediction.

To my knowledge, the first in a series of downscaling experiments for European stations was conducted by von Storch et al. (1993). For this and the subsequent experiments the North Atlantic/European section of the global circulation, represented either as sea level pressure or geopotential height fields, was chosen as the predictor. von Storch et al. (1993) predicted winter mean precipitation over Spain, explaining as much as $50 \%$ of the total variance. A similar result has been obtained by Werner \& von Storch (1993), who showed that one can successfully model the mean winter temperature of Central Europe, explaining about $65 \%$ of total variance. Heyen et al (1996) modeled winter sea level height in the Baltic by the sea level pressure field, reaching overall correlations of $80 \%$. Gyalistras et al. (1994) demonstrated that this method is applicable even to mountainous regions. For a whole range of variables, among them temperature, precipitation and sunshine duration, they showed that one can explain a large amount of annual variability. The best results were obtained for temperature, with about $50 \%(30 \%)$ of the winter (summer) variance explained; about $35 \%$ $(15 \%)$ of the variance was explained for precipitation. They achieved significant improvement, especially for summer temperature, by including the global near surface temperature as a predictor field. Johannesson et al. (1995) derived climate change scenarios for the Nordic countries by applying the same method, but due to deficiencies of the GCM they used the results were somewhat vague

CCA is, by definition, symmetric with respect to $G$ and $\mathcal{L}$ so that formally the roles of $\mathcal{G}$ and $\mathcal{L}$ are interchangeable. From a dynamical point of view, however, this is counterintuitive since the forcing situation $G \rightarrow \mathcal{C}$ is essentially asymmetric. The general asymmetric, linear approach (Eq. 2) seems therefore more appropriate. All techniques which are described below are nevertheless applicable in a CCA context as well.

Besides the problems already mentioned regarding the lower short-term variability of the modeled process, there is another obstacle to applying statistical downscaling techniques to shorter time scales: the appearance of non-normal quantities. By the central limit theorem, averaged values of stationary processes tend to be normally distributed, the distribution being closer to normal the longer the length of the averaging period. On the other hand, it is interesting to note that for normally distributed quantities, if the decorrelation time is small enough the correlation is independent of the averaging period.

\section{EXPANDED DOWNSCALING}

By emphasizing the importance of short-term variability of the local processes, a method can be described for directly modeling not the single daily values but their daily covariance. Suppose the covariance of the global and local processes, $g$ and $\zeta$, are given by $\mathbf{C}_{\eta}$ and $\mathbf{C}_{\ell}$, respectively. Assuming Eq. (2), the modeled local covariance is given by the term $\mathbf{L C}_{\eta} \mathbf{L}^{\top}$ which is bilinear in $\mathbf{L}$. By modifying the conventional approach, the basic downscaling procedure can be reformulated as follows: we are seeking an operator $\tilde{\mathbf{L}}$ which minimizes the error cost function $\Gamma$ under the additional side condition that the observed local covariance is preserved. That means we must solve the constrained minimization problem

$$
\begin{aligned}
& \Gamma(\tilde{\mathbf{L}})=\min \\
& \tilde{\mathbf{L}} \mathbf{C}_{g} \tilde{\mathbf{L}}^{\mathrm{T}}=\mathbf{C}_{f}
\end{aligned}
$$

The side condition for $\tilde{\mathbf{L}}$ can be satisfied if the dimension of the global field $\mathcal{G}, n$, and that of the local field $\mathcal{L}$. $m$, obey the inequality $n \geq(m+1) / 2$. But since $G$ usually carries enough degrees of freedom this is no real limitation. Omitting the side condition gives the conventional downscaling operator $\mathrm{L}$. In the following, I will refer to all methods which use some form of Eq. (5) to estimate the global-local relation as 'conventional' downscaling, and the variance-optimized version (Eq. 6) as 'expanded' downscaling, emphasizing that 

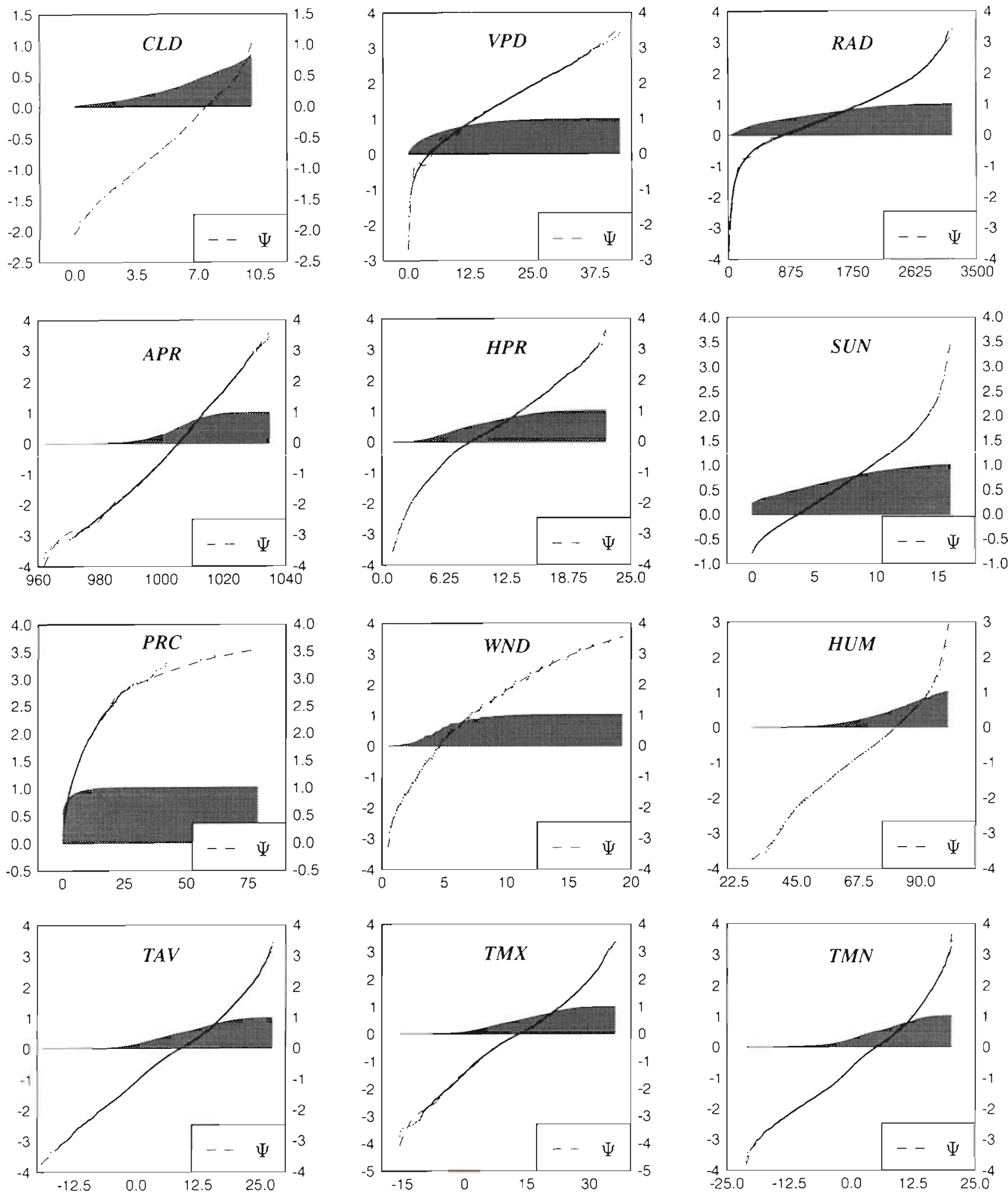

Fig. 1. Cumulative daily distributions (grey area) of 12 of the 14 Potsdam station variables (leaving out SNW and FOG) together with their probut $\Psi$ (dashed curve) as approximated by B-splines. Units given in Table 1. Temperature and pressure values appear relatively norma and the probit almost linear, whereas all moisture-related quantities, in partıcular PRC, are quite non-normal with a strongly nonlinear probit. Note, moreover, that nonlinearities are created by predefined minima and maxima, e.g. for CLD 
the reduced variances derived from $\mathbf{L}$ are expanded back to original size when applying $\dot{\mathbf{L}}$. Eq. (6) is the main equation of expanded downscaling. Having determined its solution, global and local covariance are linked by the following bilinear mapping:

$$
\mathrm{C}_{g} \mapsto \mathrm{C}_{l}=\overline{\mathrm{LC}}_{y} \tilde{\mathbf{L}}^{\top}
$$

It has to be emphasized that Eq. (7) is completely different in character from Eq. (2). The latter is meant to describe the daily relationship between global and local weather patterns whereas Eq. (7) relates weather statistics. To summarize the conditions which should be met for applications of expanded downscaling: the global circulation field, $G$, should contain enough degrees of freedom such that the constrained minimization problem Eq. (6) is uniquely solvable for a given locality $\mathcal{L}$. The mapping $(7)$ is assumed to hold for a variety of climates.

Applying Eq. (7) and feeding the resulting covariance $\mathbf{C}_{l}$ into a random number generator creates a multivariate local process ( that has the same covariance as the observed. Eq. (7) can therefore be interpreted as the basic equation for a weather generator. But it is easier to directly insert $\mathbf{L}$ into Eq. (2) and calculate $\mathbf{C}_{f}$ a posteriori. If the climate of the global process $g$ has not changed, $\mathbf{C}_{f}$ will be equal, by way of construction, to the observed covariance

Note that conventional as well as expanded statistical downscaling have at their heart the assumption that there is a (bi)linear relation between global and local weather patterns which applies to a whole variety of climates. This can be questioned since the determination of that relation requires exactly that which it is supposed to be independent of, namely the covariance of a specific climate; see Eqs. (5) \& (7).

The validation of an expanded downscaling model uses the same approach as for the conventional case, that is, by verifying the statistical stability of the estimated covariance matrices. If this is guaranteed the modeled process covariance will, under the same climatic conditions, perfectly agree with the desired observed covariance. But note that the dependence on $\mathrm{C}_{y}$ and $\mathbf{C}_{l}$ of the operator $\mathbf{L}$ in Eq. (6) is more complicated in comparison with the conventional case (Eq. 5). This should not play a major role in well-behaved cases, but in others it might produce misleading results if the estimation is poor. To have a common measure of model performance, the quantity $\varepsilon$ which is defined in Appendix 1 is used. It is a measure that lies between correlation and 'explained variance' (see e.g. Xu \& von Storch 1990) in the sense that, if the modeled amplitude is the same as the observed, $\varepsilon$ equals the former, and if it is much smaller it equals the latter.

It is desirable that the expanded $\tilde{\mathrm{L}}$ is not too different from the conventional L. Otherwise, the daily down- scaled values $\hat{i}$ would no longer be consistent with the daily global circulation $g$, with the consequence that possible shifts of scenario climate means would be incorrectly reflected at the local site. All this applies even more when one considers that the modeled signals are enhanced when the variability is greater. Scenario calculations, therefore, have to be interpreted with great care. For example, if it turns out that the local precipitation is badly modeled (as measured e.g by $\varepsilon$ ) then the signal, although strong, is just as uncertain. In a sense, only the combination of the modeled quantity and its uncertainty have objective meaning

Note that the expansion of a downscaling model is not confined to the linear case: any application of a least-squares regression method results in a damping of amplitudes. The replacement of the corresponding unconstrained error minimization problem with the constrained problem will transform the downscaling into the expanded form.

In this study we are dealing with a record / of 14 daily measurements of key meteorological quantities observed at Potsdam, Germany, since the 1890s (Table 1). This site is particularly useful as the record is almost homogeneous through this entire period, and statistical estimates should be quite stable. Fig. 1 depicts the daily distributions of $r$ as calculated between 1940 and 1989. Now it is clear that many of the distributions shown, such as those of most moisture variables (PRC, CLD, HUM; see abbreviations in Table 1), are far from being normal. It is a trivial fact that as soon as the measurement scale of a quantity includes maximum or minimum values (e.g. for WND, SUN) the quantity is bound to be non-normal. This causes the following dilemma: the predictor variables $g$ that represent the global circulation, such as sea surface pressure or geopotential height, are basically

Table 1. The 14 weather variables measured at the Potsdam station

\begin{tabular}{|lll|}
\hline Variable & Abbrev. & Units \\
\hline Temp. (mean) & TAV & ${ }^{\circ} \mathrm{C}$ \\
Temp. (max) & TMX & ${ }^{\circ} \mathrm{C}$ \\
Temp. (min) & TMN & ${ }^{\circ} \mathrm{C}$ \\
Precipitation & PRC & $\mathrm{mm}^{-1}$ \\
Wind speed & WND & $\mathrm{m} \mathrm{s}^{-1}$ \\
Rel, humidity & HUM & $\%$ \\
Air pressure & APR & $\mathrm{hPa}$ \\
Vapor pressure & HPR & $\mathrm{hPa}$ \\
Sunshine & SUN & $\mathrm{h}$ \\
Cloudiness & CLD & 1 to 10 \\
Snow height & SNW & $\mathrm{cm}$ \\
Fog & FOG & 0 to 1 \\
Vapor pressure deficit & VPD & $\mathrm{hPa}$ \\
Radiation & RAD & $\mathrm{J} \mathrm{cm}{ }^{-2}$ \\
& & \\
\hline
\end{tabular}


normal. But linear transformations leave the main characteristics (such as normality) of distributions invariant. The modeled local process $\hat{l}$ will therefore exhibit the same such characteristics as $g$, and will thus be normal. The discrepancy between the distributions of $\hat{l}$ and $l$ would be fatal were there not a general recipe for transforming any quantity into one that is normal, and vice versa.

\section{NORMALIZING TRANSFORMATIONS: PROBITS}

It is possible to transform any unbounded, continuous random variable $X$ to a variable $Y$ which is $(0,1)$ normally distributed, i.e. normal with zero mean and unit variance. The main observation is that if the distribution function of $X$ at $X$ is $F(x)$, the new random variable $F(X)$ is uniformly $(0,1)$-distributed. From this it is clear that with the normal $(0,1)$-distribution function $\Phi(y)$, the transformations

$$
\begin{gathered}
Y=\Psi(X)=\Phi^{-1}(\mathrm{~F}(X)) \\
X=\Psi^{-1}(Y)=\Phi\left(\mathrm{F}^{-1}(Y)\right)
\end{gathered}
$$

give a one-to-one map between $X$ and the normal variate $Y$. The map $\Psi$ is often referred to as the 'probit' (= probability integral transformation) of $X$. For more on probits see e.g. Appendix 2 or Ledermann (1984). In applications, however, we are dealing with distribution functions $F(x)$ which are discrete and sometimes bounded, and are thus represented by non-continuous, non-invertible step functions. To form continuous, invertible probits, statistical fits to the given discrete data are used. From Fig. 1 it can be seen that the various distribution functions span a wide range of differ- ent functional classes: from those which are almost linear and where the quantity itself is normal (such as TAV and APR) to those where the quantities are highly non-normal (such as PRC or RAD). The mapping of the end points of $X$ to $-\infty$ and $+\infty$, respectively, is particularly difficult. Thus it is necessary to choose a genuine function class for each quantity and try to estimate the necessary fit parameters (see e.g. Stidd 1973, Richardson 1977, Bardossy \& Plate 1992, Hutchinson et al. 1993 who used a power law for PRC). But this itself is worth a separate study. Instead, I chose B-splines as a uniform class of base functions. Although B-splines are entirely polynomial, the different choice of knots offers wide flexibility in the fitting. To take the annual cycle into account, I calculated the probit for each month separately (for details on B-splines refer to Appendix 2).

This still leaves the problem of bounded values, as exemplified the bound PRC $=0$. It is obvious that the image of PRC under $\Psi, Y$, must have a lower bound $y_{0}$ $=\Psi(0)$, and consequently $Y$ cannot be normal. Here, the introduction of synthetic values can help. Each time the value $\mathrm{PRC}=0$ is measured, we randomly choose, from an externally generated, normally distributed ensemble, a value $y$ such that $y \leq y_{0}$. As a result, the outcome will be a normally distributed variable in the whole range between $-\infty$ and $+\infty$. Since by the general use of B-splines the mapping of extreme values is rather poor, especially when the deviation from normality is strong, the modeling of extreme events - such as, for PRC, long, dry periods or heavy summer rainfall-should be undertaken with great caution. Work is under way to improve on the normalization step.

Table 2. Covariance matrix for the normalized station values (which is practically the correlation). Note the small correlation between temperature and pressure, and the very high positive correlations between temperature and HPR. WND and APR are positively correlated as well. PRC shows various correlations to other moisture related variables. HUM and SUN (CLD) are

\begin{tabular}{|c|c|c|c|c|c|c|c|c|c|c|c|c|c|c|}
\hline & TAV & TMX & TMN & PRC & WND & HUM & APR & HPR & SUN & CLD & SNW & $\mathrm{FOG}$ & VPD & RAD \\
\hline TAV & 1.0 & 0.9 & 0.8 & 0.0 & 0.1 & -0.3 & -0.1 & 0.7 & 0.2 & -0.1 & -0.2 & 0.0 & 0.5 & 0.2 \\
\hline TMX & & 1.0 & 0.7 & -0.1 & 0.0 & -0.4 & 0.0 & 0.6 & 0.4 & -0.3 & -0.2 & -0.1 & 0.7 & 0.4 \\
\hline TMN & & & 1.0 & 0.1 & 0.1 & 0.0 & -0.2 & 0.8 & -0.1 & 0.1 & -0.2 & 0.0 & 0.3 & -0.1 \\
\hline PRC & & & & 1.0 & 0.2 & 0.4 & -0.4 & 0.2 & -0.4 & 0.4 & 0.0 & 0.0 & -0.4 & -0.4 \\
\hline WND & & & & & 1.0 & -0.1 & -0.3 & 0.0 & 0.0 & 0.0 & 0.0 & -0.2 & 0.0 & -0.1 \\
\hline HUM & & & & & & 1.0 & -0.2 & 0.3 & -0.7 & 0.6 & 0.0 & 0.2 & -0.8 & -0.7 \\
\hline APR & & & & & & & 1.0 & -0.2 & 0.3 & -0.3 & 0.0 & 0.0 & 0.1 & 0.3 \\
\hline HPR & & & & & & & & 1.0 & -0.2 & 0.2 & -0.1 & 0.1 & 0.1 & -0.2 \\
\hline SUN & & & & & & & & & 1.0 & -0.8 & 0.0 & -0.1 & 0.7 & 0.9 \\
\hline CLD & & & & & & & & & & 1.0 & 0.0 & 0.1 & -0.6 & -0.7 \\
\hline SNW & & & & & & & & & & & 1.0 & 0.0 & -0.1 & 0.0 \\
\hline FOG & & & & & & & & & & & & 1.0 & -0.2 & -0.1 \\
\hline VPD & & & & & & & & & & & & & 1.0 & 0.7 \\
\hline RAD & & & & & & & & & & & & & & 1.0 \\
\hline
\end{tabular}
strongly negatively (positively) correlated 
Fig. 2. Time series of TMX (lower panel) and PRC (upper panel) from observations (OBS), conventional downscaling (DS) and expanded downscaling (EDS) for summer 1968. Note the good correspondence on the weekly time scale for TMX. The monthly anomaly amplitudes seem to be correct. For PRC, no clear weekly variability can be seen. With the exception of mid June, the clustering of PRC events corresponds to observations, but the large event in early May, possibly from a thunderstorm, has no counterpart in the model
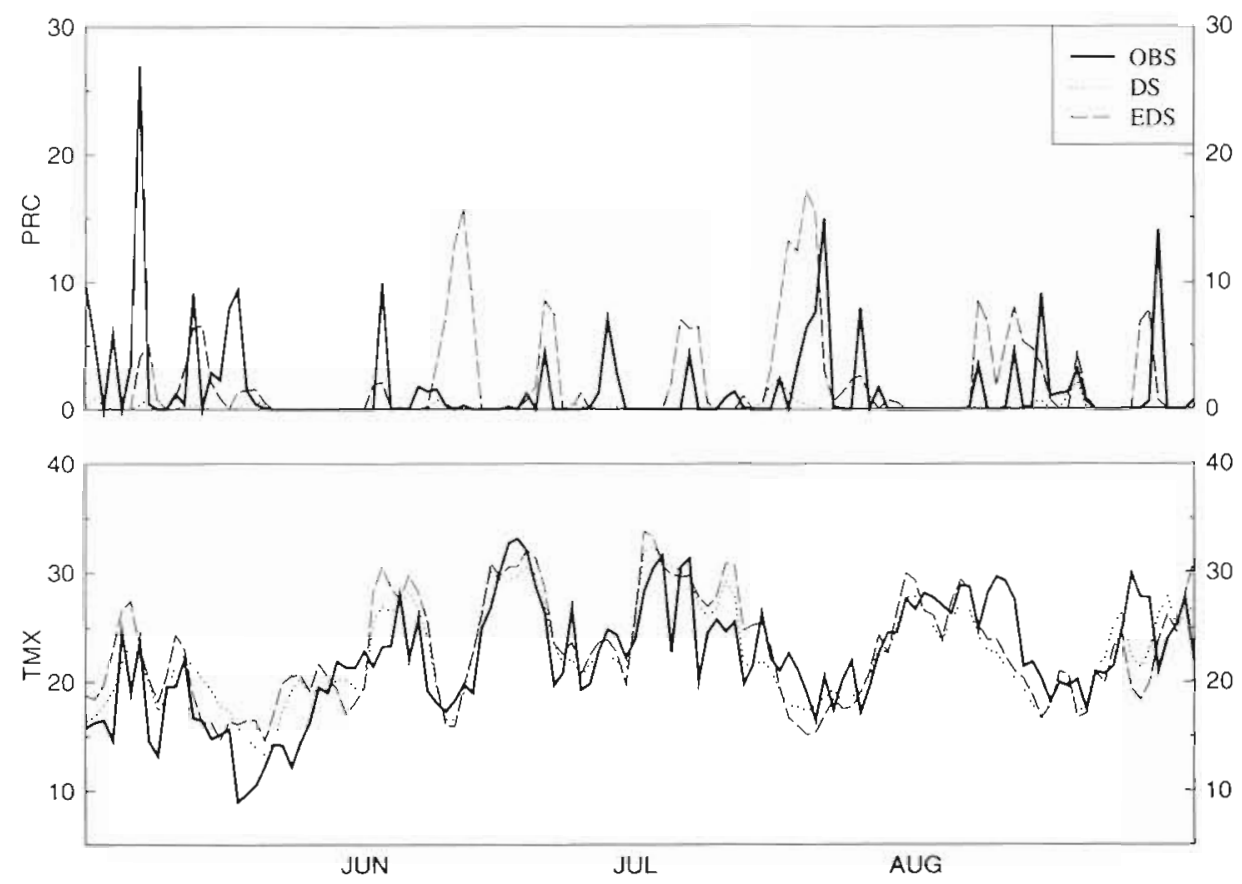

To check their interdependence, I calculated the daily covariance (= correlation) matrix of the 14 probitnormalized variables. The result is shown in Table 2. Note the strong correlation between temperature and HPR. Generally there are only low correlations between PRC and any of the other variables; only for A.PR and partly for SUN do we find a consistent anticorrelation with PRC. During summer, TMX seems to dominate many other variables (such as HUM, HPR, SUN, CLD, VPD, RAD). This annual correlation does not resolve possible seasonal cycles. A more detailed seasonal calculation (not shown) revealed, for example, a significant seasonal cycle in the correlation between temperature and wind, starting with positive correlations in winter that decreased and became negative through spring and summer, and increased again towards fall and winter. By definition, expanded downscaling is meant to preserve exactly this structure of variance and interdependence of the local variables unless the global covariance has changed as the result of a climate change.

\section{DOWNSCALING THE NORTH ATLANTIC CIRCULATION}

A good representation of the large scale atmospheric circulation provides, among other variables, the $500 \mathrm{hPa}$ geopotential height field (z500). Daily z500 maps exhibit mostly large-scale structures at least $1000 \mathrm{~km}$ in extent. Furthermore, compared to other, more complex fields, the $z 500$ field is relatively well modeled by current atmospheric general circulation models (AGCMs). Hence it is only natural to choose this field as a predictor. Furthermore, it gives the best prediction results. I used daily analyses provided by the National Center of Atmospheric Research (NCAR) in 1992 (see Mass et al. 1987) from which I selected the Northern Atlantic and European regions in the longitude-latitude box between $55^{\circ} \mathrm{W}-25^{\circ} \mathrm{N}$ and $45^{\circ} \mathrm{E}-$ $75^{\circ} \mathrm{N}$. To reduce the noisy components the fields were projected onto the first few EOFs explaining $90 \%$ of the daily variance about their long-term annual average. For model calibration, the period 1 January 1975 to 31 December 1989 was chosen; all fitting was done separately for the 4 seasons. Validation was done using the period 1 October 1962 to 31 December 1974

The 14 leading EOFs of the $z 500$ field explained $90 \%$ of the daily variance. Using the 14-dimensional vector of the corresponding principal components as the predictor we have enough degrees of freedom ( 8 would be sufficient) to allow expanded downscaling to be applied. Fig. 2 shows a typical outcome of the experiment for summer 1968; it shows observed and downscaled values for TMX and PRC, for both conventional and expanded downscaling. Note the very good agreement, for both methods, between the TMX curves on the weekly time scale. On the other hand, the expanded curve clearly shows enhanced variability. For precipitation this has dramatic effects: whereas there is practically no conventionally downscaled precipitation, the expanded version appears much more realistic. There are certainly large daily errors. But when we look at the clustering of rainfall events, such as those in early May, 

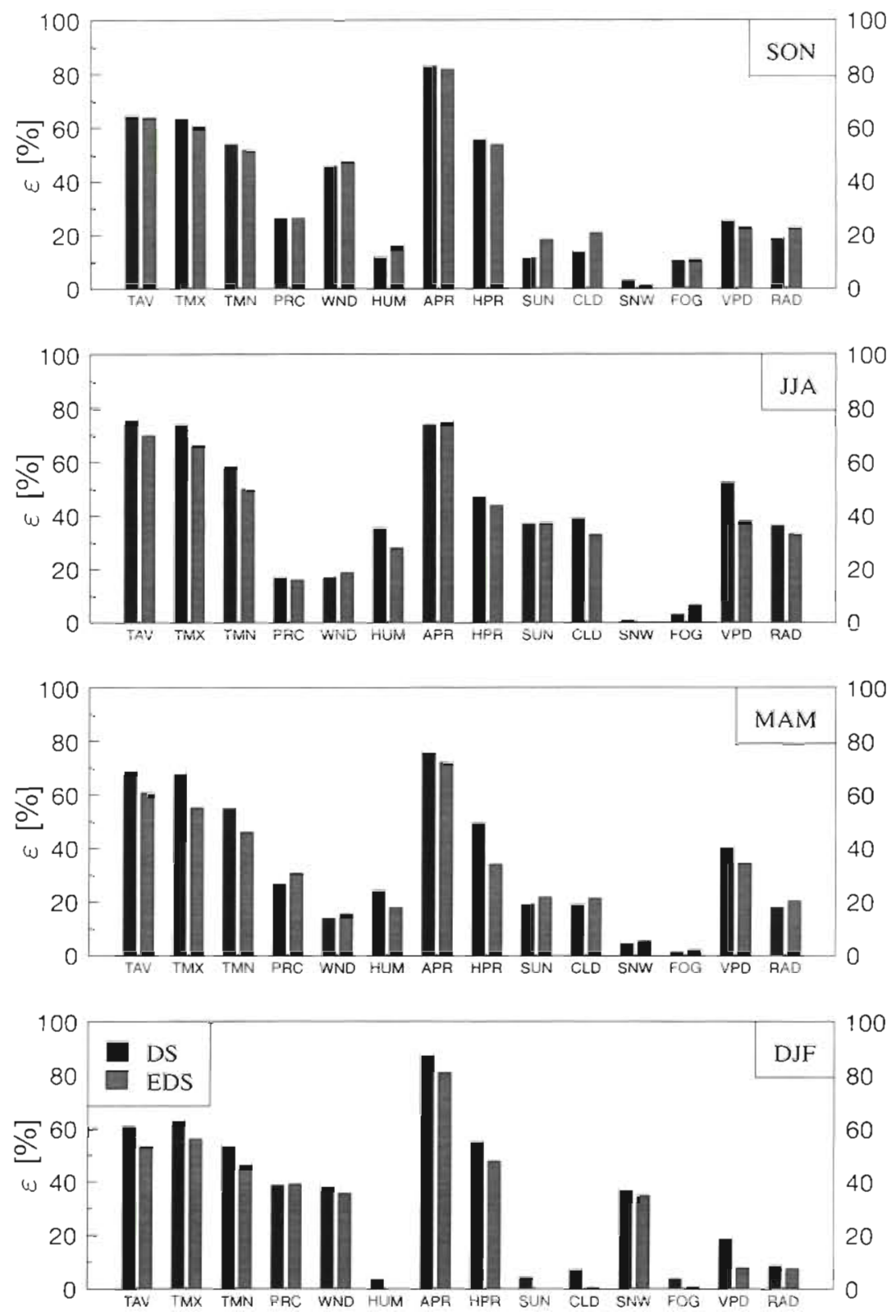

Fig. 3. Performance, expressed in values of $\varepsilon$, of the conventional downscaling (DS) and expanded downscaling (EDS) for the Potsdam variables, for each season. The predictor is the observed $z 500$ field over the North Atlantic and Europe. The period for calibration was 1975-89, and for validation 1962-74. Note the large differences betwern the 'easy' variables such as APR and the 'hard' moisture quantities (PRC, HI ' $M$, CLD). Apart from winter all EDS values are positive

normalization step may be required. On the other hand, the information that is contained in the global $z 500$ field (in summer) is probably not enough to derive local thundershowers from it. The inclusion of the 850 hPa temperature field, for instance, as a predictor might help in this regard.

As a measure of how strongly the conventional downscaling operator has been modified through the optimization, I calculated the model performance $\varepsilon$ of daily values of the normalized variables for both operators. The result is shown in Fig. 3. Note that, since for the optimized operator the modeled amplitude equals the observed, the $\varepsilon$ values are simply correlations. Clearly, for all seasons and for conventional as well as expanded downscaling, the variable APR (air pressure) is best predicted with $\varepsilon$ as high as $85 \%$, followed by the temperature variables with $\varepsilon$ at about 70 to $75 \%$. Here, and in most other cases, the minimization reduces $\varepsilon$ by less than 5 to $10 \%$, depending on the season. Generally, all highly nonlinear (non-normal), moisture-related quantities such as precipitation, cloudiness and sunshine have much smaller values, reaching at most $40 \%$ for winter precipitation and summer cloudiness. Large seasonal variability is observed in the wind variable: here we have spring and summer values of about $15 \%$ but in summer/fall this increases to over $40 \%$. It is interesting to note that the expanded operator sometimes even produces higher scores than the conventional, as can be seen e.g. in spring precipitation.

For the winter season, not much confidence can be put into the estimated amounts of HUM, SUN, CLD and FOG. For the rest of the year, all $\varepsilon$ scores are well above 0 for the

late July and mid-August, it can be concluded that they are quite well represented. Singular events, such as the heavy rainfall that was observed early May and which was probably caused by a thundershower (see TMX), or the strong model event in mid-June, are not well matched. Generally, the non-normal clustering is still stronger in the observed rainfall compared to the model. This indicates that further improvement in the expanded case, and this is statistically significant due to the long period of estimation (12 seasons $\approx 1000 \mathrm{~d}$ per season). But although a correlation of $20 \%$, say, may not be too meaningful, it at least points to the fact that the modeled quantities are consistent with the daily values of $g$, the global field. This will be of importance for the interpretation of downscaling from AGCM scenarios. 
As has been mentioned, the inclusion of other predictors promises to improve the model's predictive skill. But it might have other merits: since the seasonal cycle is perhaps better represented in those other fields (consider e.g. temperature), the model's dependence on the climatological seasonal cycle can be relaxed.

\section{THE ECHAM SCENARIOS}

There is no need to give a comprehensive introduction to the ECHAM AGCM; see Cubasch et al. (1992) or Roeckner et al. (1992). There exist a number of versions of the model which differ in physical schemes as well as in the chosen resolution. Two $30 \mathrm{yr}$ runs of ECHAM3 with T42 resolution, as described in Perlwitz \& Graf (1996), will be used here. One is a control run with climatological ocean boundary conditions and the other was performed as a time-slice experiment with modified oceanic conditions. The oceanic conditions, i.e. sea surface temperatures, are determined from a T21 ECHAM1 run coupled to an OGCM loceanic GCM), onto which increasing greenhouse gas forcing has been imposed according to the IPCC scenario A emissions. The mean ocean state of the first decade of that run is taken as a boundary for the control run, whereas the final decade is taken for the scenario run.

To apply the downscaling we select, from each daily ECHAM 2500 field, the Northern Atlantic/European area as described above, and interpolate the model grid onto the coarser observational grid (using bilinear interpolation). Since we are dealing with an anomaly model it is relevant which climatology we use for calculating the field anomalies. Like in most other scenario downscaling applications, for the IPCC scenario A run we treat the model climatology, i.e. the annual cycle produced by the control run, as a basic reference, and calculate monthly anomalies relative to that cycle. Here we assume that the climate change signal is unaffected, as a first approximation, by the model climatology. On the other hand, we want to keep the anomalies as small as possible. In this way the error that is fed to the nonlinear parts of the model (i.e. normalization) is minimized. The resulting anomaly field will be referred to as SCA. The model errors relative to the observed climatology are denoted by CTL, and they will, like SCA, be interpreted as a scenario. Fig 4 shows the seasonal means of the CTL and SCA anomaly fields. CTL performs relatively poorly during winter, with a strong positive anomaly of up to $120 \mathrm{~m}$ over the Northern Mediterranean and a negative anomaly of $-30 \mathrm{~m}$ over Greenland. The Mediterranean anomaly roughly corresponds to an average high sealevel pressure system of about $1020 \mathrm{hPa}$, and thus can- not be neglected; over the year the anomaly weakens and has a minimum of about $60 \mathrm{~m}$ (over the Atlantic) in summer. Another negative anomaly can be seen over the Baltic States (spring) and Finland (summer). But since this is rather small and probably not so influential for our area of interest, we can ignore it. Under SCA conditions the $z 500$ height field is increased throughout the year and almost over the entire area, the only exception being a $z 500$ reduction in spring off the coast of England. The strongest positive anomalies appear over Southern Europe. A high pressure ridge builds up during spring, forming 2 separate cells over Spain and the Black Sea in summer, and matures in fall, with maxima of $90 \mathrm{~m}$ over those cells. The geostrophic wind which can be deduced from the SCA signal is such that the high pressure ridge over the Mediterranean deflects the westerly jet stream northward. Hence, the inflow of moist Atlantic disturbances onto the European continent, the main source of precipitation, is interrupted. In winter, apart from an intensification over Eastern Europe, no significant signal can be identified compared to the CTL error.

Using expanded downscaling we can now infer 2 scenarios for the locality of Potsdam, which will also be called CTL and SCA. It is certainly desirable that the CTL scenario be as close to observations as possible; only then can we have enough confidence in a modeled local quantity to derive climate change information. The cumulative distribution functions of the normalized variables are depicted in Fig. 5. Not only do these results affirm that application of Eq. (2) creates normally distributed variables, even for PRC and CLD, but we can even see clear profiles of the scenario A signal, especially in the fall season. Here, for example, less humidity and enhanced sunshine lead to higher temperatures. The signal persists in the winter season, but here the modelling is not as good. In spring, the warming is weaker and the humidity is increased, similarly to the summer season.

After rescaling via the inverse probit (see Eq. 8b), we can calculate explicit amplitudes of the various scenarios. However, for many variables the exact outcome is of Iittle value, and the results cannot serve as much more than a check of model consistency. Fig. 6 depicts the monthly means for the various variables. The clearest signal is again the temperature increase throughout the year, especially as seen in TMN and TMX. In fall, TMX shows values that are about 3 to $5^{\circ} \mathrm{C}$ higher. In summer and fall this is caused by stronger radiative forcing due to enhanced sunshine. Note the large discrepancies in the control climate for several moisture variables (such as PRC in summer or CLD all year). Therefore, one should not put too much faith in these results, with a possible exception of HPR, the vapor pressure. Moisture is too nonlinear/non-normal 

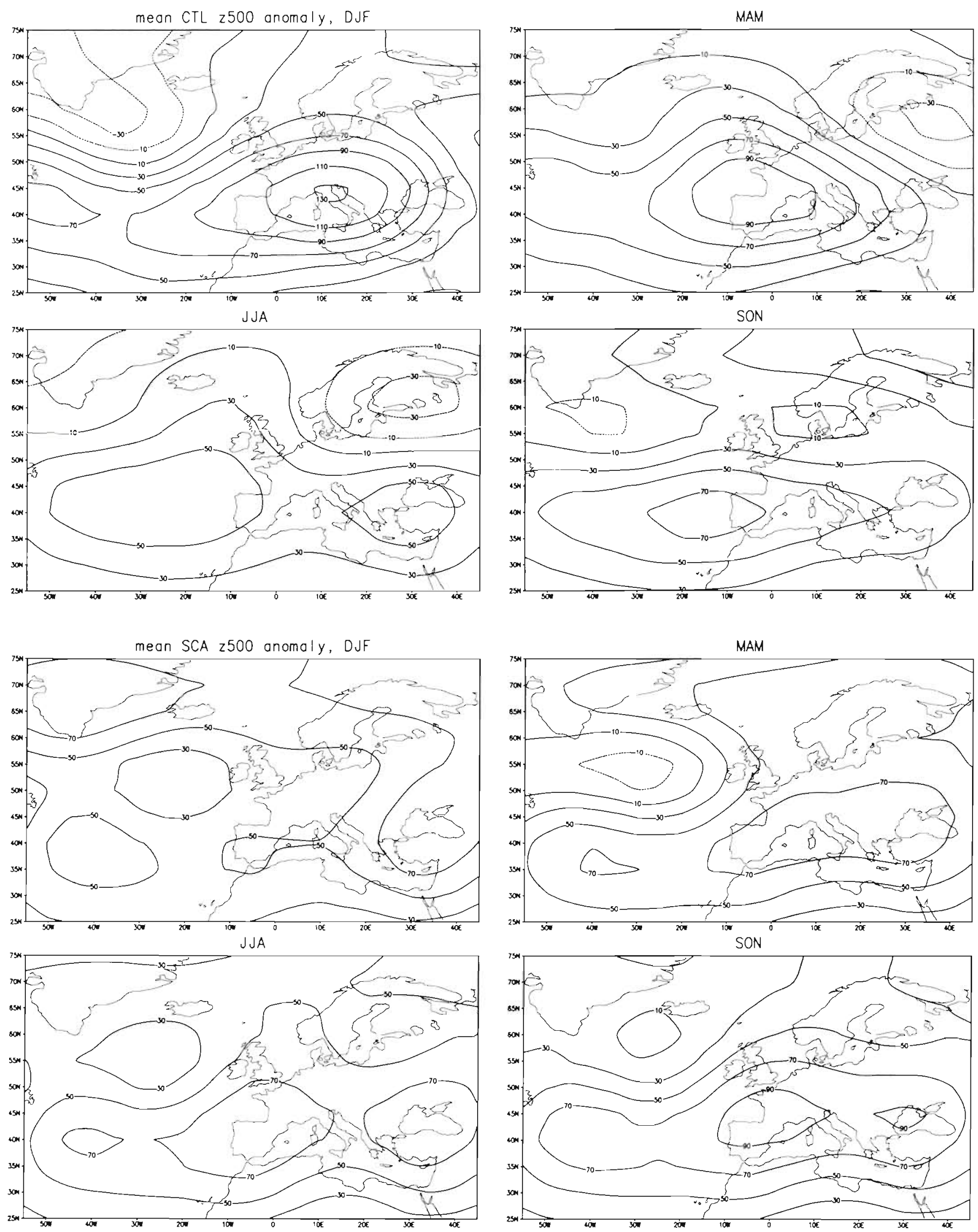

Fig. 4. Seasonal means of the CTL and SCA z500anomaly fields, in meters. Note the poor performance of the control run (CTL) in winter and spring. The SCA signal is positive throughout, with maximum amplitudes during summer and fall over Spain and the Black Sea 
(a) DJF
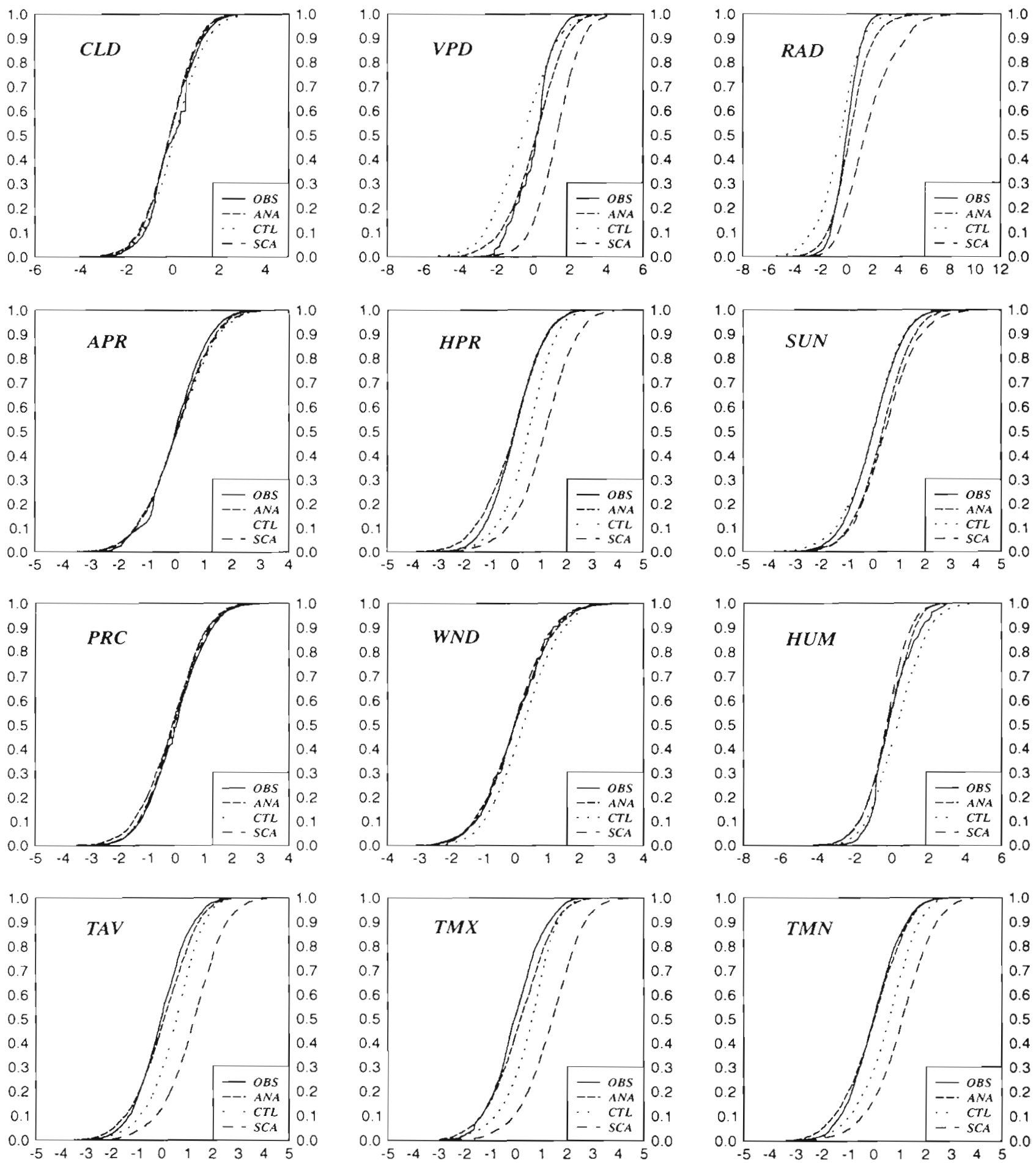

Fig. 5 (above and on following pages). Cumulative distribution functions of the normalized Potsdam station variables (without SNW and FOG), as shown by observations (OBS), the control scenario (CTL) and scenario A (SCA). (a) Winter. All 3 temperature quantities are increased in scenario $A$, together with increased SUN, VPD and RAD values

a quantity to allow specific and safe estimates to be derived from the GCM. Due to the strong nonlinearities in the PRC probit this might just be the effect of a smaller daily variability of ECHAM under SCA conditions, as is indicated by Fig. 5. This vagueness is even greater in summer where, in SCA, the 
(b) MAM
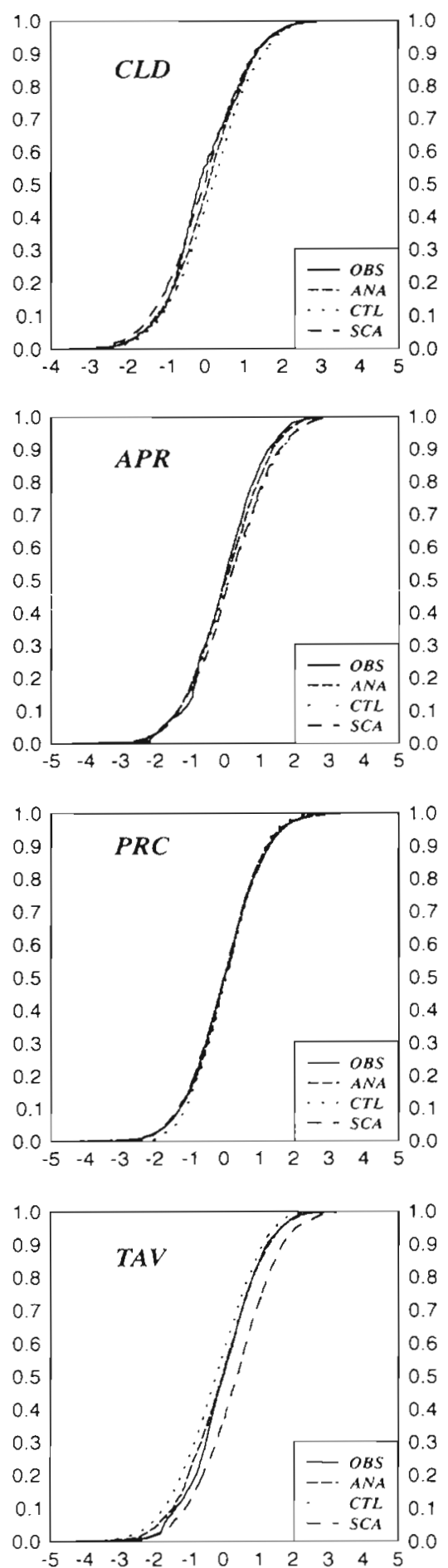
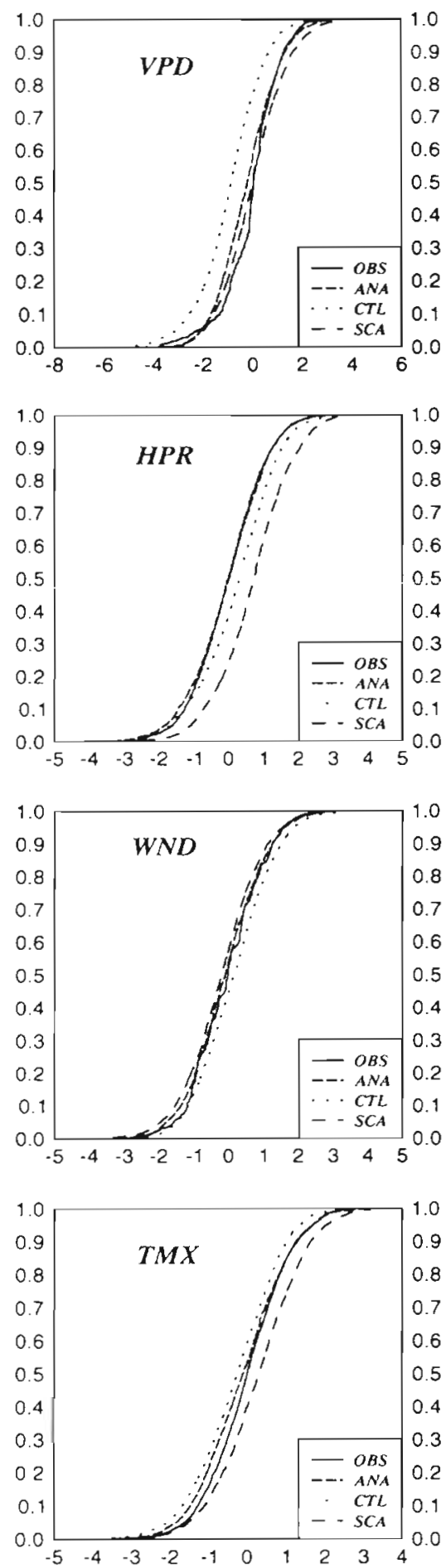
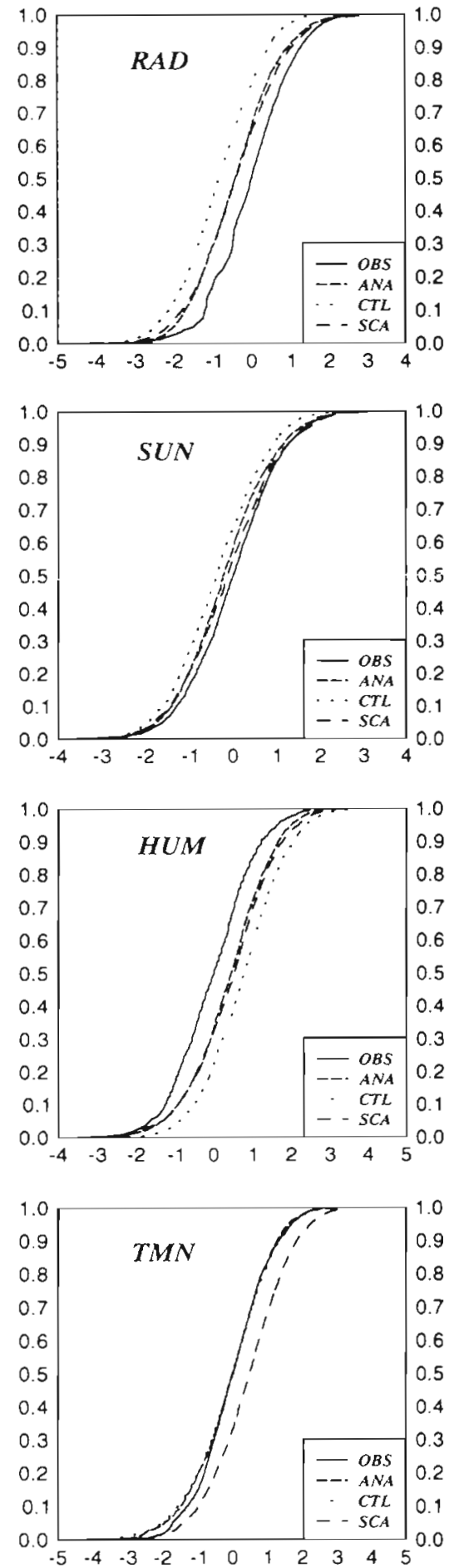

Fig. 5, continued. (b) Spring. The main signal is a slight temperature increase (TAV, TMX) and greater humidity

PRC and HPR signals are positive while CLD clearly decreases. This seeming contradiction, however, can possibly be resolved by considering the opposite influence that hot and humid summertime conditions have on CLD and PRC means, noting the strong effect of thunderstorms. Nevertheless, there is much room for improvement of the moisture quantities, which are so important. 
(c) JJA
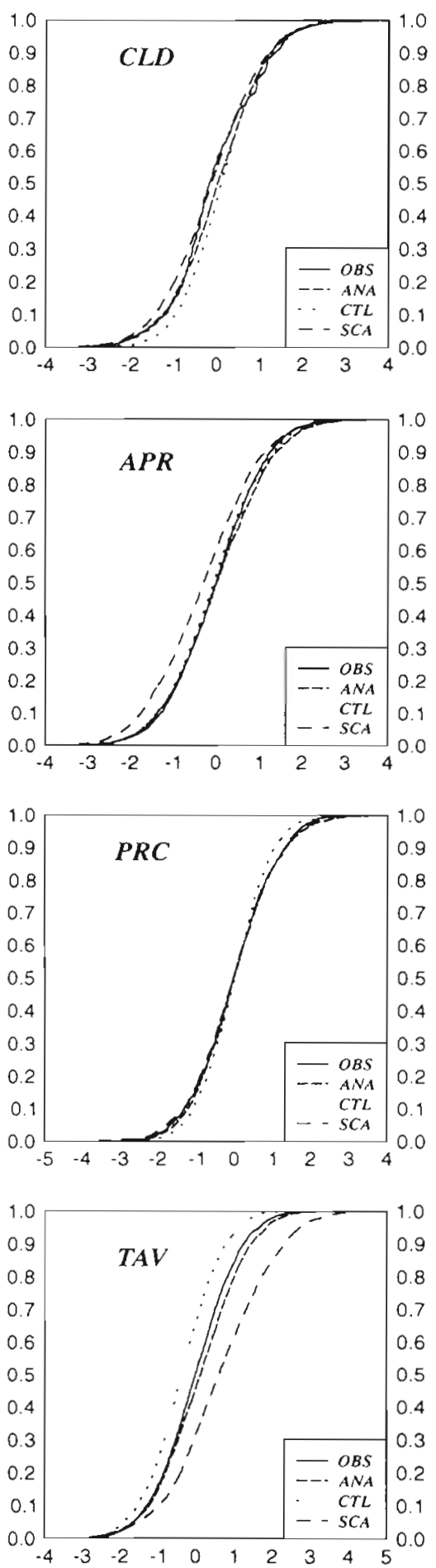
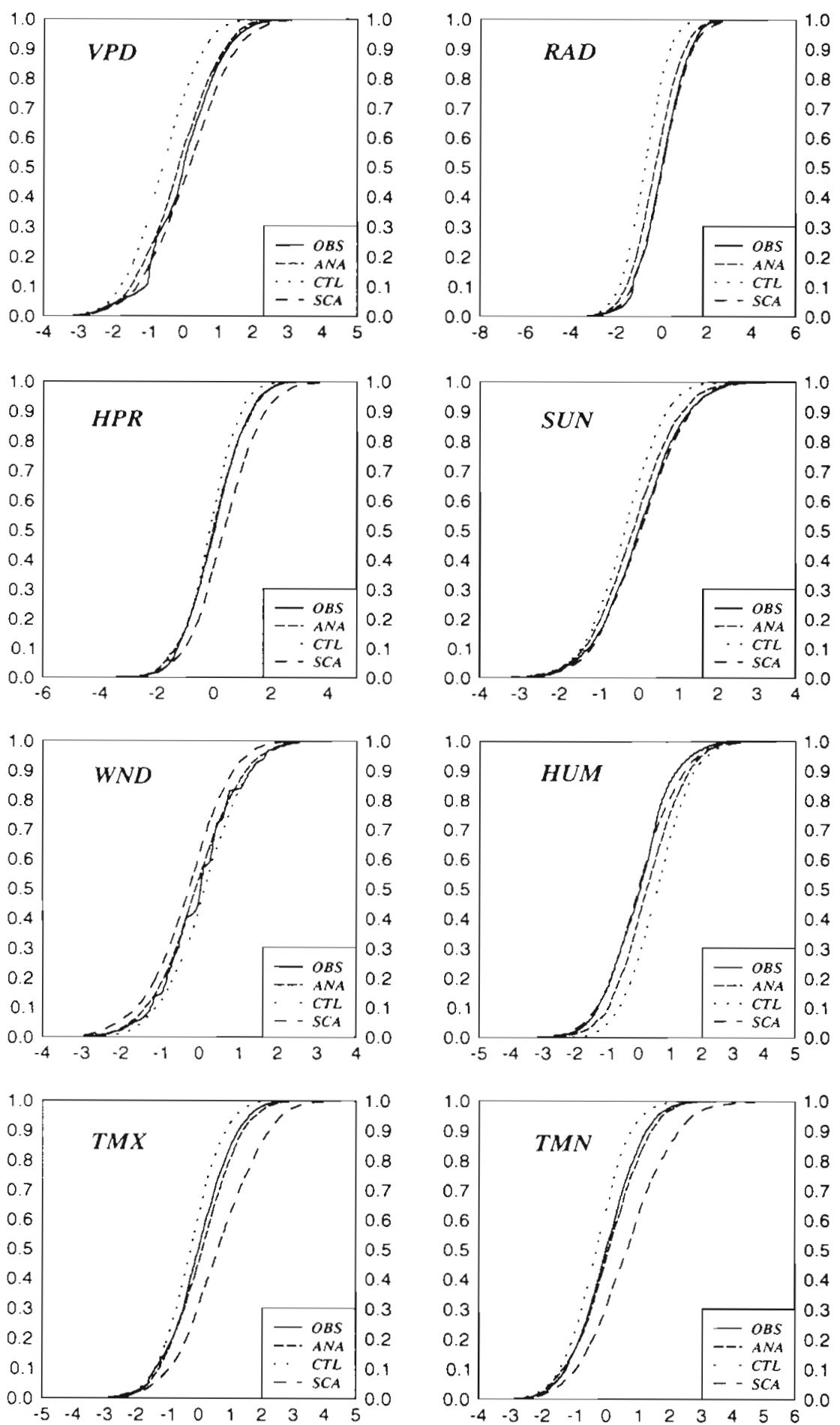

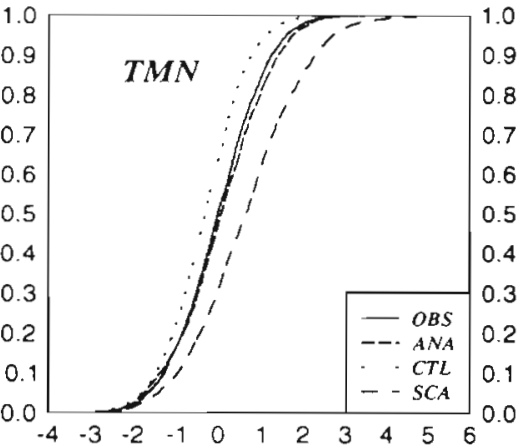

Fig. 5, continued. (c) Summer. Marked temperature increase and lower winds. Enhanced HPR and weaker APR

\section{CONCLUSIONS}

Current downscaling techniques attempt to estimate possible shifts in regional or local climate conditions from GCM runs forced with certain greenhouse gas concentrations. Although there are a growing number of quite satisfying results, there is an inherent general drawback, in particular at the interface to ecosystem 
(d) $\mathrm{SON}$
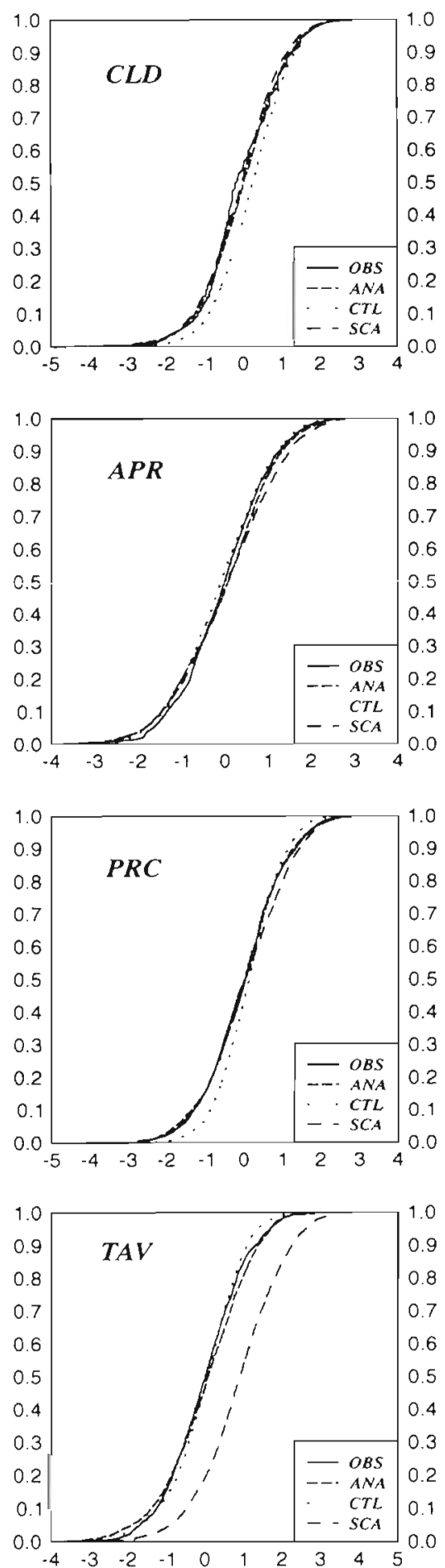
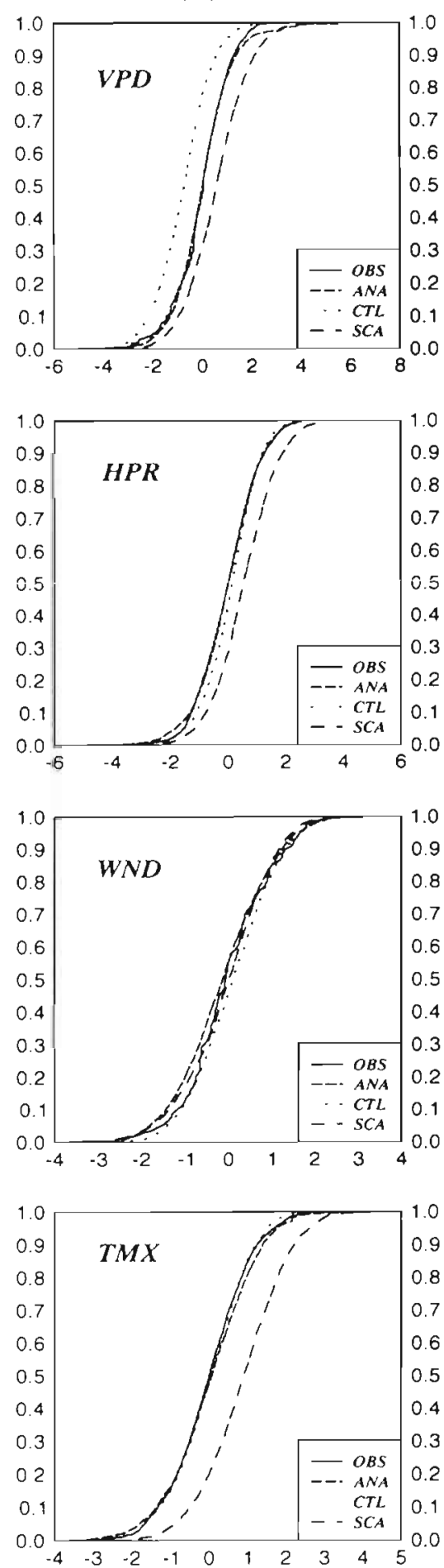
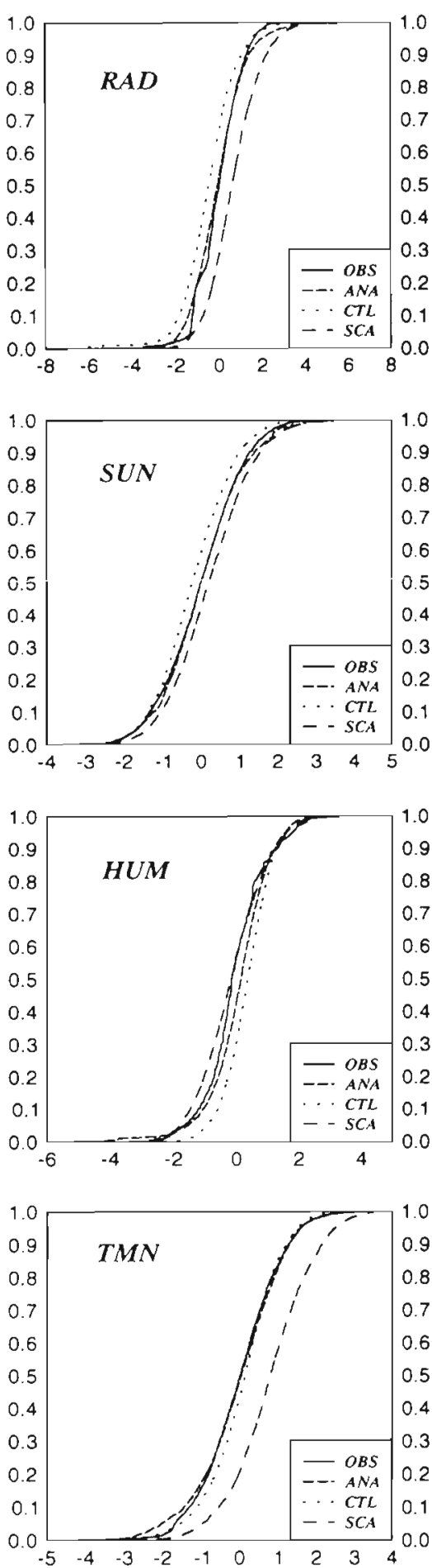

Fig. 5, continued. (d) Fall. Together with a significant temperature increase there are indications of enhanced sunshine (see SUN, VPD and RADI

modeling, of statistical models as long as they are built on some form of regression: no realistic short-term variability can be expected, as the amplitudes of the modeled processes are generally too low. This is grounded in the fact that all regression models are 'conservative' in the following sense: if only a little information can be gained from the learning procedure, the model returns a process of small amplitude. 
응

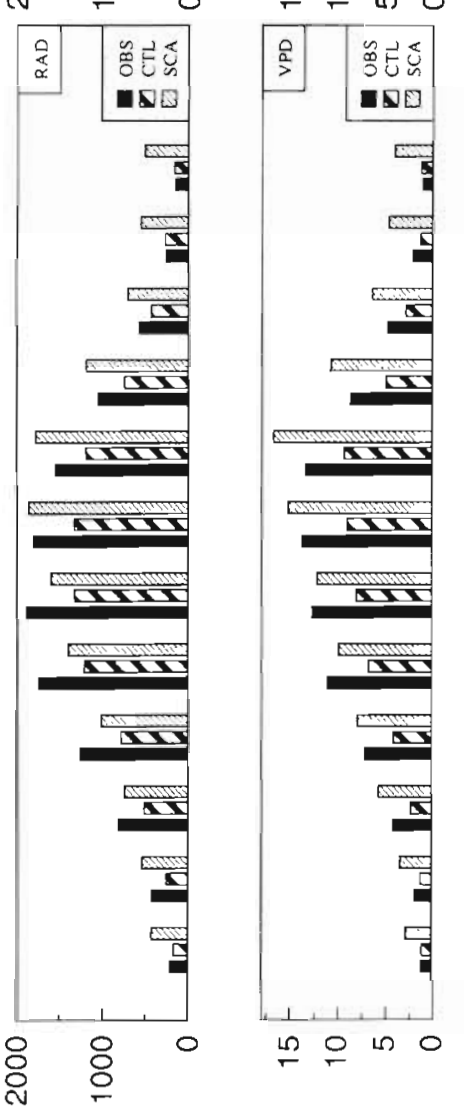

용

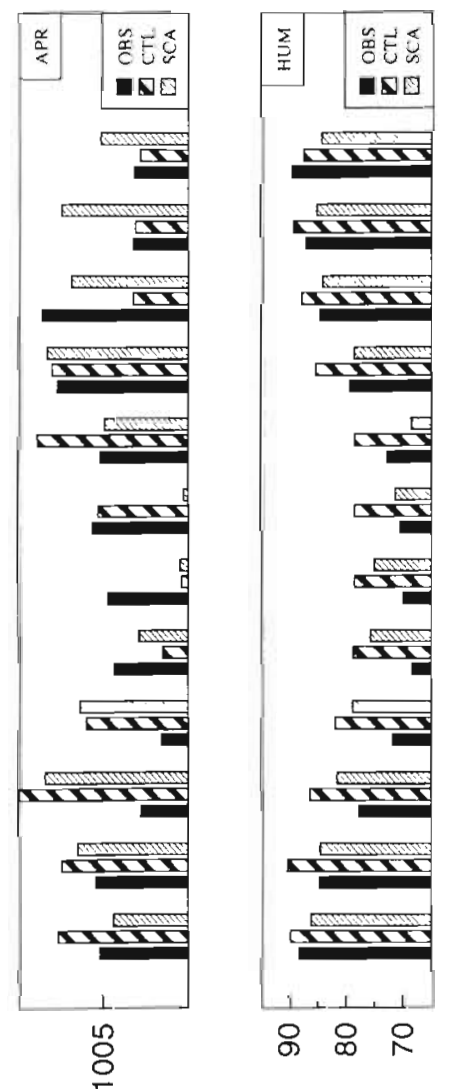

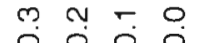

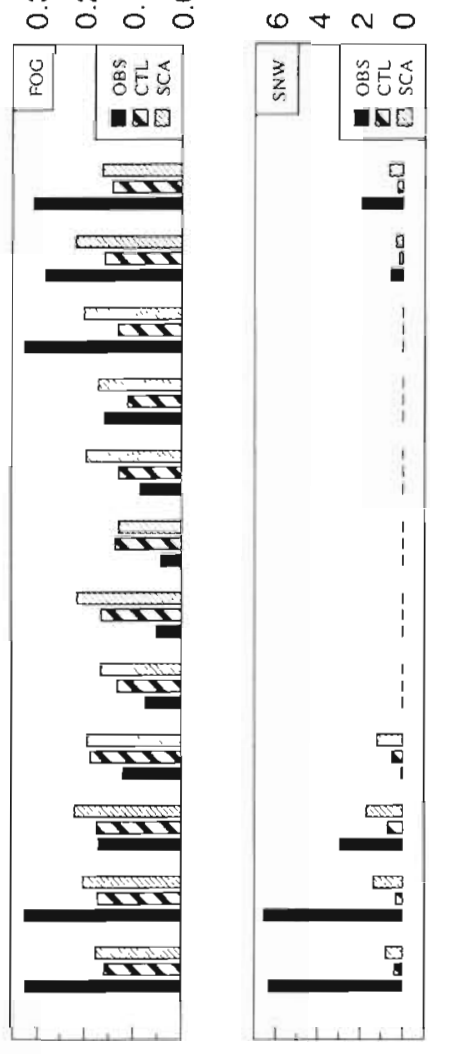

m $N-0$

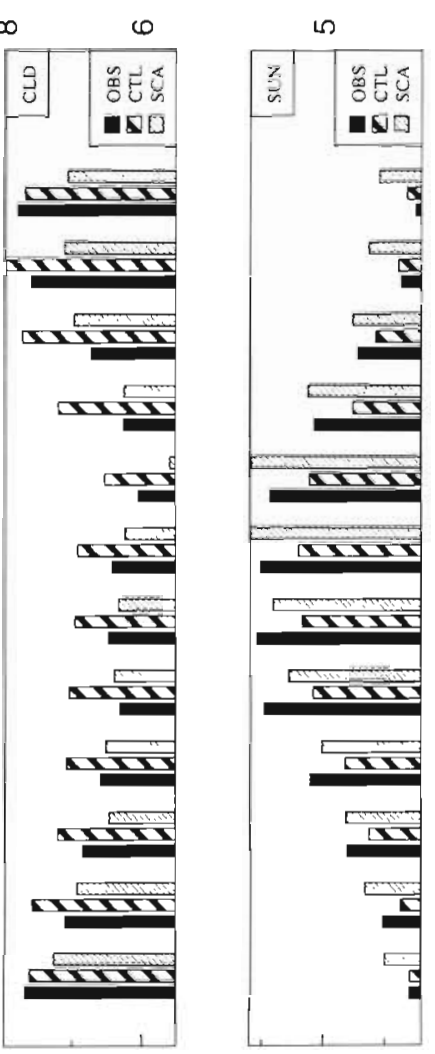

เ

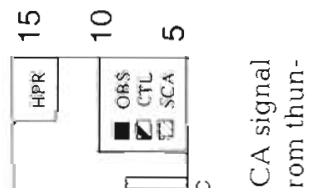

प은

믐

2 के के

का $\stackrel{5}{ \pm}$

… त

때용 \&

खण⿵ है है

पार्य के के

凶以

음

$\Sigma 00$.

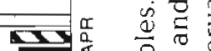

ए人,

(1) 0 :

ए㟧

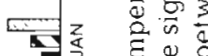

ए)

运运

응

$\widetilde{0}$

2

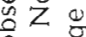

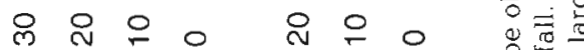

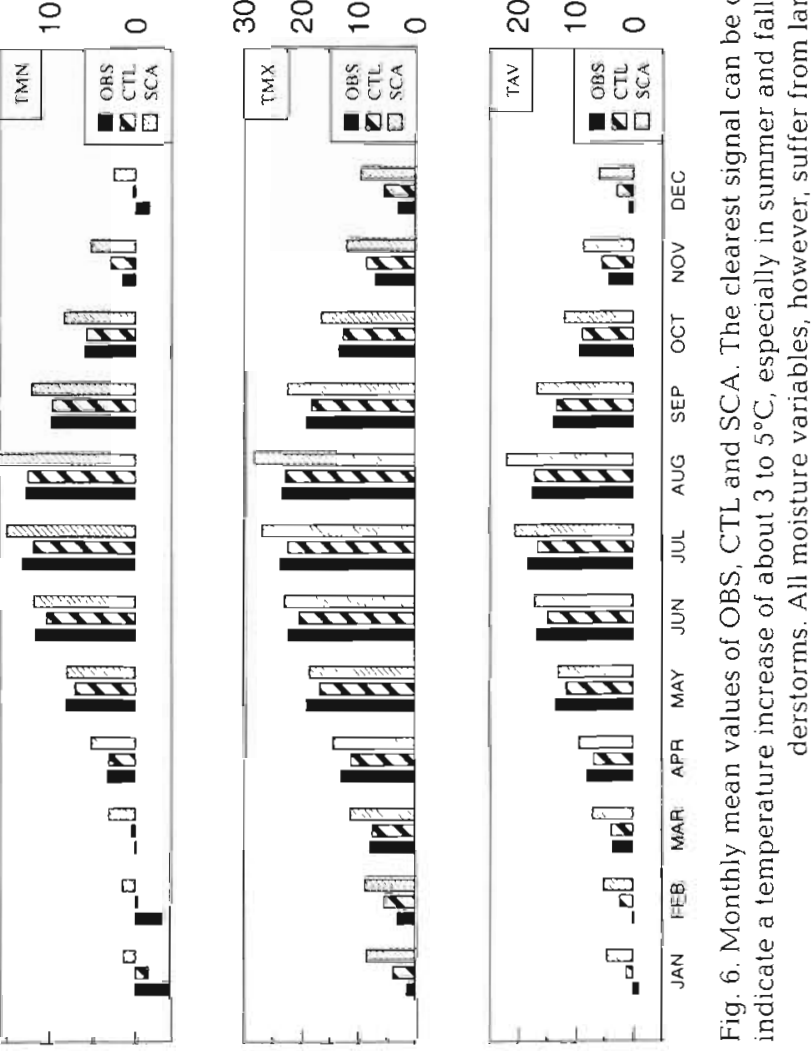

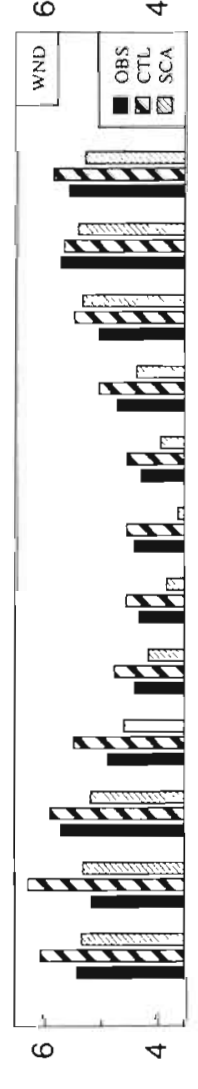

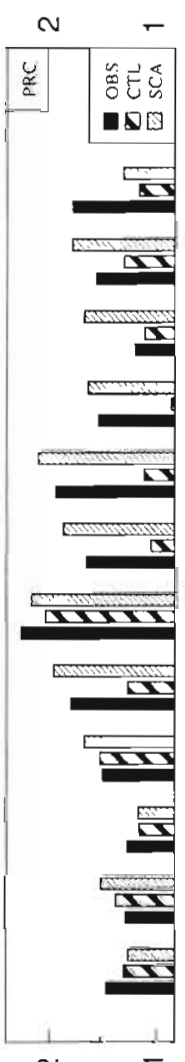

오응

$\therefore \circ 0$ 
More precisely, the ratio of amplitudes of the modeled and the observed process is directly bounded above by the correlation between the predictor and predictand field. Even in the case of high correlations this can undermine ecosystem modeling considerably, since many systems require the whole range of climate variability in order to fully function. On the other hand, a realistic short-term statistic is all that many of these models really need from the climatic side. This is probably the reason why regression models, which includes all linear statistical downscaling models, have so far been applied only to long-term means and their possible change under emission scenarios. Furthermore, modeling short-term variability via regression appears to involve taking a detour since one would, in a first step, estimate the daily or weekly values themselves and then, in a second step, estimate from the result the short-term statistics.

In my approach I have tried to model mean and short-term variability directly: the covariance of the global circulation is linked to the local covariance of a weather site in a bilinear way. Regression models usually proceed by minimizing an error cost function that depends on the transfer-function parameters. In expanded downscaling, this is turned into a constrained minimization by the requirement that the local covariance be preserved. The degree of modification that the unconstrained, classical solution undergoes is directly linked to the quality of that solution: the smaller the error the less it gets modified. But the direction of modification is, of course, not towards a smaller error but towards the original covariability, the price being that the expected error is increased. In a way, the dominance of global-local correlation is replaced by a dominance of local-local correlations.

A solution to the constrained minimization exists if the global dimension exceeds a certain limit depending on the local dimension. The resulting expanded downscaling operates on the global covariance and outputs a local covariance which, in turn, can be used to generate random numbers and local weather scenarios. As such, expanded downscaling appears to be a combination of regression models and weather generators. But it is not necessary to generate numbers: direct application of the expanded operator to the global fields produces a local process which has the 'correct' covariance. Furthermore, correlations that prevailed between predictor and predictand are only slightly disturbed so that global information is correctly passed to the modeled process, just like in regression models. Hence, the means themselves are modeled as accurately as possible.

Since the predictor fields are predominantly normally distributed the same is true, by linearity, for the predictands. This requires a one-to-one normalization. for which I chose a general B-spline approximation of the probits. It is clear that this choice, although rather general, is not perfect and should be refined for highly non-normal quantities such as precipitation. Since modeling of extreme values is particularly bad for nonnormal quantities, and the modeled process retains its original variability as much as possible, this leads to large model errors. Consequently, only modeled values and model error information together give a complete picture of a local scenario.

In 3 experiments I predicted 14 meteorological quantities at the Potsdam weather station from the North Atlantic/European atmospheric circulation, as represented by the $500 \mathrm{hPa}$ geopotential height field. The first experiment used analyzed fields to calibrate and validate the model. This turned out to model normalized pressure and temperature values quite satisfactorily. For temperature, for example, this means that the overall weekly variability was represented quite well by the model. Compared to the conventional linear regression model, the variability of the expanded downscaling model was visibly closer to the observations. This became particularly clear in the case of precipitation: whereas the regression model was bound to produce almost zero variability (due to the low correlation), expanded downscaling was capable of detecting precipitation clusters correctly and returning realistic precipitation sums. As it seems, the only error was: that it usually did not hit the right day. It is clear that the erroneously created rainfall events by definition produced an overall error which was larger compared to the regression model.

Using the ECHAM AGCM, the remaining 2 experiments applied expanded downscaling to a control run and a run with greenhouse gas forcing according to IPCC emission scenario $A$. In both experiments, the interplay of AGCM and downscaling errors had many unforeseeable consequences, especially for the moisture quantities, which are so important for ecosystem modeling. Because of nonlinearity and non-normality effects for those variables, even small errors in climatology and variability of the AGCM were intensified by the downscaling to climate change signal levels, and yielded unrealistic and inconsistent results. This applies in particular to the winter and summer season. In winter, the ECHAM control climate showed large discrepancies to the observations. In summer, there were indications that strong convective rainfall events were only poorly modeled. For the other seasons, a general warming trend produced by a calmer, drier, and sunnier climate was found.

As an interesting alternative to the above scenarios, especially for applications to ecosystems, the following experiment is suggested: instead of using the control climate as a reference for the global scenario A anom- 
alies, the scenario A climatology itself could be used. This extracts from the full signal everything that comes purely from a change in distributions of global variables. Expanded downscaling seems particularly suited for mapping this change of global covariance to the local scale, creating a scenario that solely reflects, in a consistent way, a possible redistribution of weather elements under climate change, while keeping the means constant.

As we have seen, there is much room for improvement. The inclusion of other predictor variables, such as $850 \mathrm{hPa}$ temperature, is one possibility. Another is the extension to more than 1 modeled site. In fact, I expect expanded downscaling to be particularly useful when applied to a whole group of weather stations.
The consistent spatial information provided by the measurements from all those sites can be comprised into a single covariance which is then used directly for the downscaling The downscaling produces fields of variables the structure and consistency of which can be analyzed much more comprehensively. Provided the obtained fields are smooth enough, further downscaling by interpolation can be done to provide the ecosystem modeler with consistent, fine-scale climate data. This can improve in particular the modeling of stratified precipitation where the large-scale covariance between stations comes into play. But it will not solve the problem of convective precipitation which occurs on the smallest scale. It is very likely that this marks the limit of all linear, or bilinear, downscaling techniques.

Appendix 1. Calculating model performance

Given an anomaly process $x(t)$ and another process $y(t)$ which is meant to model $x(t)$, a measure of model performance which is often used is the explained variance, defined as the term

$$
1-\frac{\left\langle(y-x)^{2}\right\rangle}{x^{2}}
$$

However, this has the disadvantage of deviating considerably from the classical correlation. A closer connection is achieved by taking instead the measure

$$
\varepsilon=1-\frac{\left\langle(y-x)^{2}\right\rangle}{\left\langle x^{2}\right\rangle+\left\langle y^{2}\right\rangle}
$$

For small $\left\langle y^{2}\right\rangle$ both terms coincide, and it is easy to see that under the condition of equal variance, i.e. $\left\langle x^{2}\right\rangle=\left\langle y^{2}\right\rangle \cdot \varepsilon$ is nothing other than a correlation.

Appendix 2. Normalizing via probits

Given a random variable $X$ with distribution function $F$ and a number $n$ of realizations of $X, X=\left(x_{1}, \ldots, x_{n}\right)$, and the cumulative normal distribution function $\Phi$, we approximate the probit function $Y=\Phi^{-1} F(X)$ by using B-splines in the following way. Without loss of generality we have $x_{i} \leq x_{j}$ for $i \leq j$. Now for a number $m \leq n$, let $n=m k+r$ with $r<n$. This defines a subdivision of the interval $\left[x_{1}, x_{n}\right]$ into $m$ pieces each of which contains $k$ or $r$ members of $\left(x_{1}, \ldots, x_{n}\right)$. Those pieces form the nodes of the B-splines. The data points are given by the pairs $\left(x_{1}, y_{1}\right), \ldots,\left(y_{n}, x_{n}\right)$, with $y_{j}$ denoting $\mathrm{F}\left(x_{j}\right)$ for $j \leq n$. In order to avoid overshooting effects at the boundaries 1 chose the order of the B-splines to be not more than 4 . It turned out that 12 was generally a good value for $m$. Furthermore, before calculating the B-splines I introduced new, extrapolated data which extended beyond the boundaries. For each side 5 data points, $\left(x_{1}, y_{1}\right)^{1}, \ldots,\left(x_{5}, y_{5}\right)^{\prime}$ and $\left(x_{1}, y_{1}\right)^{u}, \ldots,\left(x_{5}, y_{5}\right)^{u}$, were created according to, for each $k \leq 5$,

$$
\begin{aligned}
& x_{k}^{1}=x_{1}-k\left(x_{2}-x_{1}\right) \\
& y_{k}^{1}=x_{1}-k\left(y_{2}-y_{1}\right) \\
& x_{k}^{u}=x_{5}-k\left(x_{n}-x_{n-1}\right) \\
& y_{k}^{u}=y_{5}-k\left(y_{n}-y_{n-1}\right)
\end{aligned}
$$

This linear extrapolation has the effect that, if the quantity $X$ is strongly non-normal, extreme events of $X$ will be 'smoothed out' by the model. If the approximation is good, the B-splines define a monotone function $\hat{\psi}$ which smoothly embeds the inter$\operatorname{val} \mathcal{D}_{x}=\left[x_{5}^{1}, x_{5}{ }^{\mu}\right]$ into the reals, $R$.

$$
\hat{\psi}: D_{x} \rightarrow R
$$

If the domain of $X$ is $R, \hat{\psi}$ approximates the probit $\psi$ of $X$. The image of $X$ under $\hat{\psi}$ will therefore be approximately normally $(0,1)$-distributed. The inverse probit, $\psi^{-1}$, is approximated by the same method, where the roles of $x_{1}$ and $y_{1}$ are exchanged.

Generally, $\hat{\psi}$ maps intervals onto intervals. If the domain of $X$ is not $\mathrm{R}$ but a sequence of intervals, this method also works although the resulting image of $X$ is certainly not normally distributed. This normalization can, nevertheless, still be achieved. One merely has to replace each instance of an interval boundary by an appropriate random number. 


\section{LITERATURE CITED}

Bardossy A, Plate E (1992) Space-time model for daily rainfall using atmospheric circulation patterns. Water Resour Res $28: 1247-1259$

Barnett TP (1991) The interaction of multiple time scales in the tropical climate system. J Clim 1:269-85

Cubasch U, Hasselmann K, Höck H, Reimer EM, Mikolajewicz U, Santer B, Sausen R (1992) Time-dependent greenhouse warming computations with a coupled oceanatmosphere model. Clim Dyn 8:55-69

Elsner JB, Tsonis AA (1992) Nonlinear predictions, chaos and noise. Bull Am Meteorol Soc 78:49-60

Giorgi F (1990) Simulation of regional climate using a limitedarea model nested in a general circulation model. J Clim 3: $941-963$

Gyalistras D, von Storch H, Fischlin A, Beniston M (1994) Linking GCM-simulated climatic changes to ecosystem models: case studies of statistical downscaling in the Alps. Clim Res 4:167-189

Heyen H, Zorita E, von Storch H (1996) Statistical downscaling of monthly mean North Atlantic air-pressure to sea level anomalies in the Baltir Sea. Tellus A (in press)

Hughes JP, Lettenmaier DP, Guttorp P (1993) A stochastic approach for assessing the effect of changes in regional circulation patterns on local precipitation. Water Resour Res 29:3303-3315

Hutchinson MF, Richardson CW, Dyke PT (1993) Normalization of rainfall across different time steps. Reprinted from: Management of irrigation and drainage systems. Sponsored by the Irrigation and Drainage Division, ASCE, held 21-23 July, 1993, Park City, Utah, p $432-439$

Johannesson T, Jonsson T, Källen E, Kaas E (1995) Climate change scenarios for the Nordic countries. Clim Res 5: $181-195$

Ledermann W (ed) (1984) Handbook of applicable mathematics A, VI. Wiley \& Sons, Chıchester

Editor: H. von Storch, Geesthacht, Germany
Mass CF, Edmon HJ, Friedman HJ, Cheney NR, Recker EE (1987) The use of compact discs for the storage of large meteorological and oceanographic data sets. Bull Am Meteor Soc 68:1556-1558

Perlwitz J, Graf HF (1996) Greenhouse warming experiments with the ECHAM3-model using the time-slice method for $2 \times \mathrm{CO}_{2}$ and $3 \times \mathrm{CO}_{2}$. Report of the Max-Planck-Institut für Meteorologie, Hamburg

Richardson CW (1977) A model of stochastic structure of daily precipitation over an area. Colorado State University, Fort Collins, Hydrology Paper 91

Roeckner E, Arpe K, Bengtsson L, Brinkop S, Dümenil L, Esch $M$, Kirk E, Lunkeit $F$, Ponater $M$, Rockel B, Sausen $R$, Schleese U, Schubert S, Windelband M (1992) Simulation of the present-day climate with the ECHAM model impact of model physics and resolution. Report 93, MaxPlanck-Institut für Meteorologie, Hamburg

Stidd CK (1973) Estimating the precipitation climate. Water Resour Res 9:1235-1241

von Storch H, Zorita E, Cubasch U (1993) Downscaling of global climate change estimates to regional scales: an application to Iberian rainfall in wintertime. J Clim 6 , $1161-1171$

Werner PC, von Storch H (1993) Interannual variability of central European mean temperature in January-February and its relation to large-scale circulation. Clim Res 3: $195-207$

Wilson LL, Lettenmaier DP, Skyllingstad E (1992) A multiple stochastic daily precipitation model conditional on largescale atmospheric circulation patterns. J Geophys Res 97 : 2791-2809

Xu JS, von Storch H (1990) Predicting the state of the southern oscillation using principal oscillation pattern analysis. J Clim 3:1316-1329

Zorita E, Hughes J, Lettenmaier D, von Storch H (1995) Stochastic characterization of regional circulation patterns for climate model diagnosis and estimation of local precipitation. J Clim 8:1023-1042

Manuscript first received: December 20, 1995

Revised version accepted: May 23, 1996 